International Journal of Pure and Applied Mathematics

Volume 92 No. 5 2014, 757-776

ISSN: 1311-8080 (printed version); ISSN: 1314-3395 (on-line version)

url: http://www.ijpam.eu

doi: http://dx.doi.org/10.12732/ijpam.v92i5.10

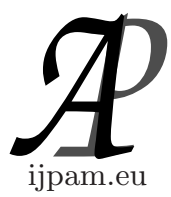

\title{
SOLVING COUPLED SYSTEM OF NONLINEAR PDE'S USING THE NATURAL DECOMPOSITION METHOD
}

\author{
Mahmoud S. Rawashdeh ${ }^{1}$, Shehu Maitama ${ }^{2}$ \\ ${ }^{1}$ Department of Mathematics and Statistics \\ Jordan University of Science and Technology \\ P.O. Box 3030, 22110, Irbid, JORDAN \\ ${ }^{2}$ Department of Mathematics and Statistics \\ Jordan University of Science and Technology \\ P.O. Box 3030, 22110, Irbid, JORDAN
}

\begin{abstract}
In this research paper, we develop a new method called the Natural Decomposition Method (NDM) for solving coupled system of nonlinear partial differential equations (CSNLPDEs). The new method is a combination of the Natural Transform Method (NTM) and the Adomian Decomposition Method $(\mathrm{ADM})$. By using the new method, we successfully handle some class of coupled system of nonlinear partial differential equations (CSNLPDEs) in a simple and elegant way. The proposed method gives an exact or approximate solution in the form of a rapid convergence series. Hence, the Natural Decomposition Method (NDM) is an excellent mathematical tool for solving linear and nonlinear differential equation. Thus, NDM can easily be used to solve a wide class of nonlinear partial differential equations (NLPDEs) and obtain an exact or analytical solution.
\end{abstract}

AMS Subject Classification: 35Q61, 44A10, 44A15, 44A20, 44A30, 44A35, $81 \mathrm{~V} 10$

Key Words: natural decomposition method, Adomian polynomials, coupled system of nonlinear partial differential equations

Received: March 14, 2014

(C) 2014 Academic Publications, Ltd.

$\S$ Correspondence author url: www.acadpubl.eu 


\section{Introduction}

Many applications in physics are modeled by nonlinear partial differential equations (NLPDEs). Numerous researchers are willing to understand these models, they insist in finding explicit exact or approximate solutions using different methods. In recent years, many researchers became interested in solving differential equations (ODEs, PDEs) and integral equations. Also, many authors have paid attention in studying the solutions of nonlinear partial differential equations by various methods.

There are many integral transform methods [3], [4], [5], [6], [7], [8], [9] and [10] exists in the literature to solve PDEs, ODEs and integral equations. The most used one is the Laplace transformation [24]. We will discuss a new integral method called the Natural Decomposition Method (NDM) and apply it to find an exact solutions to coupled systems of nonlinear PDEs. Other methods used recently to solve PDEs and ODEs and among these are; the Sumudu transform [10] and the Elzaki transform [3], [4], [5], [6], [7] and [8]. Fethi Belgacem and R. Silambarasan [14] and [15] used the N-Transform to solve the Maxwell's equation, Bessel's differential equation and linear and nonlinear Klein Gordon Equations and more. Also, Zafar H. Khan and Waqar A. Khan [17] used the NTransform to solve linear differential equations and they presented a table with some properties of the N-Transform of different functions. Moreover, they applied the N-Transform to find solution for the fluid flow problem. Finally, they proved that the N-Transform converges to the Laplace and Sumudu transform.

We present several applications in the fields of Physics and Engineering to show the efficiency and the accuracy of the NDM. The Adomian decomposition method (ADM) [1] and [2], proposed by George Adomian, has been applied to a wide class of linear and nonlinear PDEs. For the nonlinear models, the NDM shows reliable results in supplying exact solutions and analytical approximate solutions that converges rapidly to the exact solutions.

Many numerical methods were used recently to solve NLPDEs and integral equations, such as, the Adomian Decomposition Method (ADM) [1] and [2], the Reduced Differential Transform Method (RDTM) [20], [21], [22] and [23], and the Variational Iteration Method (VIM) [25].

In this paper, we solve the following CSNLPDEs: First, consider the coupled system of nonlinear partial differential equation of the form:

$$
\begin{array}{r}
v_{t}-v_{x x}-2 v v_{x}+(v w)_{x}=0 \\
w_{t}-w_{x x}-2 w w_{x}+(v w)_{x}=0,
\end{array}
$$


subject to the initial conditions

$$
\begin{aligned}
v(x, 0) & =\sin (x) \\
w(x, 0) & =\sin (x) .
\end{aligned}
$$

Secondly, we next consider the systems of nonlinear coupled partial differential equation of the form:

$$
\begin{aligned}
p_{t}+v_{x} w_{y}-v_{y} w_{x} & =-p \\
v_{t}+w_{x} p_{y}+p_{x} w_{y} & =v \\
w_{t}+p_{x} v_{y}+p_{y} v_{x} & =w,
\end{aligned}
$$

subject to the initial conditions

$$
\begin{aligned}
p(x, y, 0) & =e^{x+y} \\
v(x, y, 0) & =e^{x-y} \\
w(x, y, 0) & =e^{y-x} .
\end{aligned}
$$

The rest of this paper is organized as follows: In Section 2 and 3, the NDM and $\mathrm{ADM}$ are introduced. In Section 4, we explain the methodology of the NDM. Section 5 is devoted to apply the NDM to two test coupled systems to show the effectiveness of our method. Section 6 discussion and conclusion of this paper.

\section{Background Materials}

In this section, we present some background about the nature of the Natural Transform Method (NTM). Assume we have a function $f(t), t \in(-\infty, \infty)$, and then the general integral transform is defined as follows [11] and [12]:

$$
\Im[f(t)](s)=\int_{-\infty}^{\infty} K(s, t) f(t) d t,
$$

where $K(s, t)$ represent the kernel of the transform, $s$ is the real (complex) number which is independent of $t$. Note that when $K(s, t)$ is $e^{-s t}, t J_{n}(s t)$ and $t^{s-1}(s t)$, then Eq. (2.1) gives, respectively, Laplace transform, Hankel transform and Mellin transform.

Now, for $f(t), t \in(-\infty, \infty)$ consider the integral transforms defined by:

$$
\Im[f(t)](u)=\int_{-\infty}^{\infty} K(t) f(u t) d t,
$$


and

$$
\Im[f(t)](s, u)=\int_{-\infty}^{\infty} K(s, t) f(u t) d t .
$$

It is worth mentioning here when $K(t)=e^{-t}$, Eq. (2.2) gives the integral Sumudu transform, where the parameter $s$ replaced by $u$. Moreover, for any value of $n$ the generalized Laplace and Sumudu transform are respectively defined by [11] and [12]:

$$
\ell[f(t)]=F(s)=s^{n} \int_{0}^{\infty} e^{-s^{n+1} t} f\left(s^{n} t\right) d t,
$$

and

$$
\mathbb{S}[f(t)]=G(u)=u^{n} \int_{0}^{\infty} e^{-u^{n} t} f\left(t u^{n+1}\right) d t .
$$

Note that when $n=0$, Eq. (2.4) and Eq. (2.5) are the Laplace and Sumudu transform respectively.

\section{Definitions and Properties of the N-Transform}

The natural transform of the function $f(t)$ for $t \in(-\infty, \infty)$ is defined by [11] and [12]:

$$
\mathbb{N}[f(t)]=R(s, u)=\int_{-\infty}^{\infty} e^{-s t} f(u t) d t ; \quad s, u \in(-\infty, \infty),
$$

where $\mathbb{N}[f(t)]$ is the natural transformation of the time function $f(t)$ and the variables $s$ and $u$ are the natural transform variables. Note that Eq. (3.1) can be written in the form [11] and [12]:

$$
\begin{aligned}
& \mathbb{N}[f(t)]=\int_{-\infty}^{\infty} e^{-s t} f(u t) d t ; \quad s, u \in(-\infty, \infty) \\
&=\left[\int_{-\infty}^{0} e^{-s t} f(u t) d t ; \quad s, u \in(-\infty, 0)\right]+\left[\int_{0}^{\infty} e^{-s t} f(u t) d t ; s, u \in(0, \infty)\right] \\
&=\mathbb{N}^{-}[f(t)]+\mathbb{N}^{+}[f(t)]=\mathbb{N}[f(t) H(-t)]+\mathbb{N}[f(t) H(t)] \\
&=R^{-}(s, u)+R^{+}(s, u),
\end{aligned}
$$

where $H($.$) is the Heaviside function. It is worth mentioning here, if the$ function $f(t) H(t)$ is defined on the positive real axis, $t \in(0, \infty)$ and in the 
set $A=\left\{f(t): \exists M, \tau_{1}, \tau_{2}>0,|f(t)|<M e^{\frac{|t|}{\tau_{j}}}\right.$, if $\left.t \in(-1)^{j} \times[0, \infty)\right\}$, then we define the Natural transform (N-Transform) as:

$$
\mathbb{N}[f(t) H(t)]=\mathbb{N}^{+}[f(t)]=R^{+}(s, u)=\int_{0}^{\infty} e^{-s t} f(u t) d t ; \quad s, u \in(0, \infty) .
$$

Note if $u=1$, then Eq. (3.2) can be reduced to the Laplace transform and if $s=1$, then Eq. (3.2) can be reduced to the Sumudu transform.

Now we give some of the N-Transforms and the conversion to Sumudu and Laplace.

\begin{tabular}{|l|l|l|l|}
\hline$f(t)$ & $\mathbb{N}[f(t)]$ & $\mathbb{S}[f(t)]$ & $\ell[f(t)]$ \\
\hline 1 & $\frac{1}{s}$ & 1 & $\frac{1}{s}$ \\
\hline$t$ & $\frac{u}{s^{2}}$ & $u$ & $\frac{1}{s^{2}}$ \\
\hline$e^{a t}$ & $\frac{1}{s-a u}$ & $\frac{1}{1-u u}$ & $\frac{1}{s-a}$ \\
\hline$\frac{t^{n-1}}{(n-1)}, n=1,2, \ldots$ & $\frac{u^{n-1}}{s^{n}}$ & $u^{n-1}$ & $\frac{1}{s^{n}}$ \\
\hline $\sin (t)$ & $\frac{u}{s^{2}+u^{2}}$ & $\frac{u}{1+u^{2}}$ & $\frac{1}{1+s^{2}}$ \\
\hline
\end{tabular}

Table 1: Special N-Transforms and the conversion to Sumudu and Laplace

Remark: The reader can read more about the N-Transform in [17] and [19].

Some basic Theorems of the N-Transforms are given as follows [11], [12], [17] and [19]:

Theorem 1. If $R(s, u)$ is the Natural transform and $F(s)$ is the Laplace transform of the function $f(t) \in A$, then $\mathbb{N}^{+}[f(t)]=R(s, u)=\frac{1}{u} \int_{0}^{\infty} e^{-\frac{s t}{u}} f(t) d t=$ $\frac{1}{u} F\left(\frac{s}{u}\right)$.

Theorem 2. If $R(s, u)$ is the Natural transform and $G(u)$ is the Sumudu transform of the function $f(t) \in A$, then $\mathbb{N}^{+}[f(t)]=R(s, u)=\frac{1}{s} \int_{0}^{\infty} e^{-t} f\left(\frac{u t}{s}\right) d t=$ $\frac{1}{s} G\left(\frac{u}{s}\right)$.

Theorem 3. If $\mathbb{N}^{+}[f(t)]=R(s, u)$, then $\mathbb{N}^{+}[f(a t)]=\frac{1}{a} R(s, u)$.

Theorem 4. If $\mathbb{N}^{+}[f(t)]=R(s, u)$, then $\mathbb{N}^{+}\left[f^{\prime}(t)\right]=\frac{s}{u} R(s, u)-\frac{f(0)}{u}$. 
Theorem 5. If $\mathbb{N}^{+}[f(t)]=R(s, u)$, then $\mathbb{N}^{+}\left[f^{\prime \prime}(t)\right]=\frac{s^{2}}{u^{2}} R(s, u)-\frac{s}{u^{2}} f(0)-$ $\frac{f^{\prime}(0)}{u}$.

Remark: The proofs of the above theorems can be found in [11] and [12].

Remark: The Natural transform is a linear operator. That is, if $a$ and $b$ are non-zero constants, then $\mathbb{N}^{+}[a f(t) \pm b g(t)]=a \mathbb{N}^{+}[f(t)] \pm b \mathbb{N}^{+}[g(t)]=$ $a F^{+}(s, u) \pm b G^{+}(s, u)$. Moreover, $F^{+}(s, u)$ and $G^{+}(s, u)$ are the N-Transforms of $f(t)$ and $g(t)$, respectively.

\section{Analysis of the Natural Decomposition Method}

We illustrate the Natural Decomposition Method (NDM) algorithm by considering the general nonlinear non-homogeneous system of PDEs of the form:

$$
\begin{aligned}
& L_{t} v+L_{x} w+F_{1}(v, w)=h_{1}(x, t) \\
& L_{t} w+L_{x} v+F_{2}(v, w)=h_{2}(x, t),
\end{aligned}
$$

subject to the initial conditions

$$
\begin{aligned}
v(x, 0) & =g_{1}(x) \\
w(x, 0) & =g_{2}(x),
\end{aligned}
$$

where $L_{t}$ and $L_{x}$ are the first differential operators, $F_{1}(v, w), F_{2}(v, w)$ are the nonlinear operators and $h_{1}(x, t), h_{2}(x, t)$ are the nonhomogeneous terms (source terms).

We apply the N-Transform to Eq. (4.1) and Eq. (4.2) to get:

$$
\begin{aligned}
\frac{s}{u} v(x, s, u)-\frac{v(x, 0)}{u}+\mathbb{N}^{+}\left[w_{x}\right]+\mathbb{N}^{+}\left[F_{1}(v, w)\right] & =\mathbb{N}^{+}\left[h_{1}(x, t)\right] \\
\frac{s}{u} w(x, s, u)-\frac{w(x, 0)}{u}+\mathbb{N}^{+}\left[v_{x}\right]+\mathbb{N}^{+}\left[F_{2}(v, w)\right] & =\mathbb{N}^{+}\left[h_{2}(x, t)\right] .
\end{aligned}
$$

By substituting the given initials conditions in Eq. (4.2) into Eq. (4.3) we obtain:

$$
\begin{aligned}
& v(x, s, u)=\frac{g_{1}(x)}{s}+\frac{u}{s} \mathbb{N}^{+}\left[h_{1}(x, t)\right]-\frac{u}{s} \mathbb{N}^{+}\left[w_{x}+F_{1}(v, w)\right] \\
& w(x, s, u)=\frac{g_{2}(x)}{s}+\frac{u}{s} \mathbb{N}^{+}\left[h_{2}(x, t)\right]-\frac{u}{s} \mathbb{N}^{+}\left[v_{x}+F_{2}(v, w)\right] .
\end{aligned}
$$

Taking the inverse N-Transform of Eq. (4.4), we obtain

$$
v(x, t)=H_{1}(x, t)-\mathbb{N}^{-1}\left[\frac{u}{s} \mathbb{N}^{+}\left[w_{x}+F_{1}(v, w)\right]\right]
$$




$$
w(x, t)=H_{2}(x, t)-\mathbb{N}^{-1}\left[\frac{u}{s} \mathbb{N}^{+}\left[v_{x}+F_{2}(v, w)\right]\right],
$$

where $H_{1}(x, t)$ and $H_{2}(x, t)$ are the terms coming from the source terms.

Now we assume an infinite series solutions for the unknown functions $v(x, t)$ and $w(x, t)$ are given in the form:

$$
\begin{aligned}
v(x, t) & =\sum_{n=0}^{\infty} v_{n}(x, t) \\
w(x, t) & =\sum_{n=0}^{\infty} w_{n}(x, t) .
\end{aligned}
$$

Moreover, the nonlinear terms $F_{1}(v, w)$ and $F_{2}(v, w)$ can easily be decomposed as follows:

$$
\begin{aligned}
& F_{1}(v, w)=\sum_{n=0}^{\infty} A_{n}(x, t) \\
& F_{2}(v, w)=\sum_{n=0}^{\infty} B_{n}(x, t),
\end{aligned}
$$

where $A_{n}$ and $B_{n}$ are the Adomian polynomials and can easily be computed with following formulas:

$$
\begin{aligned}
& A_{n}=\frac{1}{n !} \frac{d^{n}}{d x^{n}}\left[F\left(\sum_{i=0}^{n} \lambda^{i} v_{i}\right)\right]_{\lambda=0} \\
& B_{n}=\frac{1}{n !} \frac{d^{n}}{d x^{n}}\left[F\left(\sum_{i=0}^{n} \lambda^{i} w_{i}\right)\right]_{\lambda=0},
\end{aligned}
$$

where $n=0,1,2, \ldots \quad$.

By substituting Eq. (4.8) into Eq. (4.7) we obtain:

$$
\begin{aligned}
& \sum_{n=0}^{\infty} v_{n}(x, t)=H_{1}(x, t)-\mathbb{N}^{-1}\left[\frac{u}{s} \mathbb{N}^{+}\left[\sum_{n=0}^{\infty} w_{n x}+\sum_{n=0}^{\infty} A_{n}\right]\right] \\
& \sum_{n=0}^{\infty} w_{n}(x, t)=H_{2}(x, t)-\mathbb{N}^{-1}\left[\frac{u}{s} \mathbb{N}^{+}\left[\sum_{n=0}^{\infty} v_{n x}+\sum_{n=0}^{\infty} B_{n}\right]\right] .
\end{aligned}
$$

Therefore, from Eq. (4.9) above, we can easily generate the recursive relation as follows:

$$
v_{0}(x, t)=H_{1}(x, t)
$$




$$
\begin{aligned}
& v_{1}(x, t)=-\mathbb{N}^{-1}\left[\frac{u}{s} \mathbb{N}^{+}\left[\sum_{n=0}^{\infty} w_{0 x}+\sum_{n=0}^{\infty} A_{0}\right]\right] \\
& v_{2}(x, t)=-\mathbb{N}^{-1}\left[\frac{u}{s} \mathbb{N}^{+}\left[\sum_{n=0}^{\infty} w_{1 x}+\sum_{n=0}^{\infty} A_{1}\right]\right] .
\end{aligned}
$$

Thus,

$$
v_{n+1}(x, t)=-\mathbb{N}^{-1}\left[\frac{u}{s} \mathbb{N}^{+}\left[w_{n x}+A_{n}\right]\right] n \geq 0 .
$$

Similarly,

$$
\begin{aligned}
w_{0}(x, t) & =H_{2}(x, t) \\
w_{1}(x, t) & =-\mathbb{N}^{-1}\left[\frac{u}{s} \mathbb{N}^{+}\left[\sum_{n=0}^{\infty} v_{0 x}+\sum_{n=0}^{\infty} B_{0}\right]\right] \\
w_{2}(x, t) & =-\mathbb{N}^{-1}\left[\frac{u}{s} \mathbb{N}^{+}\left[\sum_{n=0}^{\infty} v_{1 x}+\sum_{n=0}^{\infty} B_{1}\right]\right] .
\end{aligned}
$$

Eventually,

$$
w_{n+1}(x, t)=-\mathbb{N}^{-1}\left[\frac{u}{s} \mathbb{N}^{+}\left[v_{n x}+B_{n}\right]\right] n \geq 0 .
$$

Hence, the exact or approximate solutions of the nonlinear systems are given by:

$$
\begin{aligned}
v(x, t) & =\sum_{n=0}^{\infty} v_{n}(x, t) \\
w(x, t) & =\sum_{n=0}^{\infty} w_{n}(x, t) .
\end{aligned}
$$

\section{Applications}

In this section, we apply the NDM to two coupled systems and then compare our solutions to existing exact solutions.

Example 5.1. Consider the coupled system of nonlinear partial differential equations of the form:

$$
\begin{array}{r}
v_{t}-v_{x x}-2 v v_{x}+(v w)_{x}=0 \\
w_{t}-w_{x x}-2 w w_{x}+(v w)_{x}=0,
\end{array}
$$


subject to the initial conditions

$$
\begin{aligned}
v(x, 0) & =\sin (x) \\
w(x, 0) & =\sin (x) .
\end{aligned}
$$

We begin by taking the Natural transform of derivatives on both sides of Eq. (5.1) to get:

$$
\begin{aligned}
\frac{s}{u} v(x, s, u)-\frac{v(x, 0)}{u}-\mathbb{N}^{+}\left[v_{x x}\right]-2 \mathbb{N}^{+}\left[v v_{x}\right]+\mathbb{N}^{+}\left[(v w)_{x}\right] & =0 \\
\frac{s}{u} w(x, s, u)-\frac{w(x, 0)}{u}-\mathbb{N}^{+}\left[w_{x x}\right]-2 \mathbb{N}^{+}\left[w w_{x}\right]+\mathbb{N}^{+}\left[(v w)_{x}\right] & =0 .
\end{aligned}
$$

By substituting the given initial conditions in Eq. (5.2) we obtain:

$$
\begin{aligned}
v(x, s, u) & =\frac{1}{s} \sin (x)+\frac{u}{s} \mathbb{N}^{+}\left[v_{x x}+2 v v_{x}-(v w)_{x}\right]=0 \\
w(x, s, u) & =\frac{1}{s} \sin (x)+\frac{u}{s} \mathbb{N}^{+}\left[w_{x x}+2 w w_{x}-(v w)_{x}\right]=0 .
\end{aligned}
$$

Taking the inverse Natural Transform of Eq. (5.3) we obtain:

$$
\begin{aligned}
v(x, t) & =\sin (x)+\mathbb{N}^{-1}\left[\frac{u}{s} \mathbb{N}^{+}\left[v_{x x}+2 v v_{x}-(v w)_{x}\right]\right]=0 \\
w(x, t) & =\sin (x)+\mathbb{N}^{-1}\left[\frac{u}{s} \mathbb{N}^{+}\left[w w_{x x}+2 w w_{x}-(v w)_{x}\right]\right]=0 .
\end{aligned}
$$

We now assume a series solutions for the unknown functions $v(x, t)$ and $w(x, t)$ of the form:

$$
\begin{aligned}
v(x, t) & =\sum_{n=0}^{\infty} v_{n}(x, t) \\
w(x, t) & =\sum_{n=0}^{\infty} w_{n}(x, t)
\end{aligned}
$$

Then Eq. (5.5) becomes:

$$
\begin{aligned}
& v(x, t)=\sin (x)+\mathbb{N}^{-1}\left[\frac{u}{s} \mathbb{N}^{+}\left[\sum_{n=0}^{\infty} v_{n x x}+2 \sum_{n=0}^{\infty} A_{n}(v)-\left(\sum_{n=0}^{\infty} B_{n}(v, w)\right)_{x}\right] 5.7\right) \\
& w(x, t)=\sin (x)+\mathbb{N}^{-1}\left[\frac{u}{s} \mathbb{N}^{+}\left[\sum_{n=0}^{\infty} w_{n x x}+2 \sum_{n=0}^{\infty} C_{n}(w)-\left(\sum_{n=0}^{\infty} B_{n}(v, w)\right)_{x}\right]\right],
\end{aligned}
$$


where $A_{n}, B_{n}$ and $C_{n}$ are Adomian polynomials representing the nonlinear terms $v v_{x},(v w)_{x}$ and $w w_{x}$, respectively.

Then by applying the aforementioned techniques, we can generate the recursive relation as follows:

$$
\begin{aligned}
& v_{0}(x, t)=\sin (x) \\
& v_{1}(x, t)=\mathbb{N}^{-1}\left[\frac{u}{s} \mathbb{N}^{+}\left[v_{0 x x}+2 A_{0}(v)-\left(B_{0}(v, w)\right)_{x}\right]\right] \\
& v_{2}(x, t)=\mathbb{N}^{-1}\left[\frac{u}{s} \mathbb{N}^{+}\left[v_{1 x x}+2 A_{1}(v)-\left(B_{1}(v, w)\right)_{x}\right]\right] .
\end{aligned}
$$

Thus,

$$
v_{n+1}=\mathbb{N}^{-1}\left[\frac{u}{s} \mathbb{N}^{+}\left[V_{n x x}+2 A_{n}(v)-\left(B_{n}(v, w)\right)_{x}\right]\right] n \geq 0
$$

Similarly,

$$
\begin{aligned}
& w_{0}(x, t)=\sin (x) \\
& w_{1}(x, t)=\mathbb{N}^{-1}\left[\frac{u}{s} \mathbb{N}^{+}\left[w_{0 x x}+2 C_{0}(w)-\left(B_{0}(v, w)\right)_{x}\right]\right] \\
& w_{2}(x, t)=\mathbb{N}^{-1}\left[\frac{u}{s} \mathbb{N}^{+}\left[w_{1 x x}+2 C_{1}(w)-\left(B_{1}(v, w)\right)_{x}\right]\right] .
\end{aligned}
$$

Eventually,

$$
w_{n+1}=\mathbb{N}^{-1}\left[\frac{u}{s} \mathbb{N}^{+}\left[w_{n x x}+2 C_{n}(w)-\left(B_{n}(v, w)\right)_{x}\right]\right] n \geq 0
$$

Therefore, from the recursive relation derived in Eq. (5.9) and Eq. (5.11), we can compute the remaining components of the solution as follows:

$$
\begin{aligned}
v_{1}(x, t) & =\mathbb{N}^{-1}\left[\frac{u}{s} \mathbb{N}^{+}\left[v_{0 x x}+2 A_{0}(v)-\left(B_{0}(v, w)\right)_{x}\right]\right] \\
& =\mathbb{N}^{-1}\left[\frac{u}{s} \mathbb{N}^{+}\left[v_{0 x x}+2 v_{0} v_{0 x}-\left(v_{0} w_{0}\right)_{x}\right]\right] \\
& =\mathbb{N}^{-1}\left[\frac{u}{s} \mathbb{N}^{+}[-\sin (x)+2 \sin (x) \cos (x)-2 \sin (x) \cos (x)]\right] \\
& =-\sin (x) \mathbb{N}^{-1}\left[\frac{u}{s} \mathbb{N}^{+}[1]\right] \\
& =-t \sin (x) . \\
w_{1}(x, t) & =\mathbb{N}^{-1}\left[\frac{u}{s} \mathbb{N}^{+}\left[w_{0 x x}+2 C_{0}(v)-\left(B_{0}(v, w)\right)_{x}\right]\right] \\
& =\mathbb{N}^{-1}\left[\frac{u}{s} \mathbb{N}^{+}\left[w_{0 x x}+2 w_{0} w_{0 x}-\left(v_{0} w_{0}\right)_{x}\right]\right]
\end{aligned}
$$




$$
\begin{aligned}
& =\mathbb{N}^{-1}\left[\frac{u}{s} \mathbb{N}^{+}[-\sin (x)+2 \sin (x) \cos (x)-2 \sin (x) \cos (x)]\right] \\
& =-\sin (x) \mathbb{N}^{-1}\left[\frac{u}{s} \mathbb{N}^{+}[1]\right] \\
& =-t \sin (x)
\end{aligned}
$$

And

$$
\begin{aligned}
& v_{2}(x, t)=\mathbb{N}^{-1}\left[\frac{u}{s} \mathbb{N}^{+}\left[v_{1 x x}+2 A_{1}(v)-\left(B_{1}(v, w)\right)_{x}\right]\right] \\
& =\mathbb{N}^{-1}\left[\frac { u } { s } \mathbb { N } ^ { + } \left[v_{1 x x}+2\left(v_{1} v_{0 x}+v_{0} v_{1 x}\right)\right.\right. \\
& \left.\left.-\left(v_{1} w_{0}+v_{0} w_{1} x+w_{1} v_{0} x+w_{0} v_{1} x\right)\right]\right] \\
& =\mathbb{N}^{-1}\left[\frac{u}{s} \mathbb{N}^{+}[t \sin (x)-4 t \sin (x) \cos (x)+4 t \sin (x) \cos (x)]\right] \\
& =\sin (x) \mathbb{N}^{-1}\left[\frac{u}{s} \mathbb{N}^{+}[t]\right] \\
& =\sin (x) \mathbb{N}^{-1}\left[\frac{u^{2}}{s^{3}}\right] \\
& =\frac{t^{2} \sin (x)}{2 !} \text {. } \\
& w_{2}(x, t)=\mathbb{N}^{-1}\left[\frac{u}{s} \mathbb{N}^{+}\left[w_{1 x x}+2 c_{1}(w)-\left(B_{1}(v, w)\right)_{x}\right]\right] \\
& =\mathbb{N}^{-1}\left[\frac { u } { s } \mathbb { N } ^ { + } \left[w_{1 x x}+2\left(w_{1} w_{0 x}+w_{0} w_{1 x}\right)\right.\right. \\
& \left.\left.-\left(v_{1} w_{0}+v_{0} w_{1} x+w_{1} v_{0} x+w_{0} v_{1} x\right)\right]\right] \\
& =\mathbb{N}^{-1}\left[\frac{u}{s} \mathbb{N}^{+}[t \sin (x)-4 t \sin (x) \cos (x)+4 t \sin (x) \cos (x)]\right] \\
& =\sin (x) \mathbb{N}^{-1}\left[\frac{u}{s} \mathbb{N}^{+}[t]\right] \\
& =\sin (x) \mathbb{N}^{-1}\left[\frac{u^{2}}{s^{3}}\right] \\
& =\frac{t^{2} \sin (x)}{2 !} \text {. }
\end{aligned}
$$

Eventually, the approximate solution of the unknown functions $v(x, t)$ and $w(x, t)$ are given by:

$$
\begin{aligned}
v(x, t) & =\sum_{n=0}^{\infty} v_{n}(x, t) \\
& =v_{0}(x, t)+v_{1}(x, t)+v_{2}(x, t)+\cdots
\end{aligned}
$$




$$
\begin{aligned}
& =\sin (x)-t \sin (x)+\frac{t^{2} \sin (x)}{2 !}+\cdots \\
& =\sin (x)\left(1-t+\frac{t^{2}}{2 !}+\cdots\right) \\
& =e^{-t} \sin (x) .
\end{aligned}
$$

And

$$
\begin{aligned}
w(x, t) & =\sum_{n=0}^{\infty} w_{n}(x, t) \\
& =w_{0}(x, t)+w_{1}(x, t)+w_{2}(x, t)+\cdots \\
& =\sin (x)-t \sin (x)+\frac{t^{2} \sin (x)}{2 !}+\cdots \\
& =\sin (x)\left(1-t+\frac{t^{2}}{2 !}+\cdots\right) \\
& =e^{-t} \sin (x)
\end{aligned}
$$

Hence, the exact solutions of the given nonlinear coupled systems are given by:

$$
\begin{aligned}
v(x, t) & =e^{-t} \sin (x) \\
w(x, t) & =e^{-t} \sin (x) .
\end{aligned}
$$

Which is in agreement with the result obtained by HAM [26] and VIM [27].

Example 5.2. We next consider the coupled system of nonlinear PDE of the form:

$$
\begin{aligned}
p_{t}+v_{x} w_{y}-v_{y} w_{x} & =-p \\
v_{t}+w_{x} p_{y}+p_{x} w_{y} & =v \\
w_{t}+p_{x} v_{y}+p_{y} v_{x} & =w,
\end{aligned}
$$

subject to the initial conditions

$$
\begin{aligned}
p(x, y, 0) & =e^{x+y} \\
v(x, y, 0) & =e^{x-y} \\
w(x, y, 0) & =e^{y-x} .
\end{aligned}
$$

Taking the Natural Transform of derivatives on both sides of Eq. (5.19), we obtain:

$$
\frac{s}{u} p(x, y, s, u)-\frac{p(x, y, 0)}{u}+\mathbb{N}^{+}\left[v_{x} w_{y}\right]-\mathbb{N}^{+}\left[v_{y} w_{x}\right]=\mathbb{N}^{+}[-p]
$$




$$
\begin{aligned}
& \frac{s}{u} v(x, y, s, u)-\frac{v(x, y, 0)}{u}+\mathbb{N}^{+}\left[w_{x} p_{y}\right]+\mathbb{N}^{+}\left[p_{x} w_{y}\right]=\mathbb{N}^{+}[v] \\
& \frac{s}{u} w(x, y, s, u)-\frac{w(x, y, 0)}{u}+\mathbb{N}^{+}\left[p_{x} v_{y}\right]-\mathbb{N}^{+}\left[p_{y} v_{x}\right]=\mathbb{N}^{+}[w]
\end{aligned}
$$

Then by substituting the given initial conditions of Eq. (5.20) into Eq. (5.21), we have:

$$
\begin{aligned}
p(x, y, s, u) & =\frac{1}{s} e^{x+y}+\frac{u}{s} \mathbb{N}^{+}\left[v_{y} w_{x}-v_{x} w_{y}-p\right] \\
v(x, y, s, u) & =\frac{1}{s} e^{x-y}+\frac{u}{s} \mathbb{N}^{+}\left[v-w_{x} p_{y}-p_{x} w_{y}\right] \\
w(x, y, s, u) & =\frac{1}{s} e^{y-x}+\frac{u}{s} \mathbb{N}^{+}\left[w-p_{x} v_{y}-p_{y} v_{x}\right] .
\end{aligned}
$$

By taking the inverse Natural Transform of Eq. (5.22), we obtain:

$$
\begin{aligned}
p(x, y, t) & =e^{x+y}+\mathbb{N}^{-1}\left[\frac{u}{s} \mathbb{N}^{+}\left[v_{y} w_{x}-v_{x} w_{y}-p\right]\right] \\
v(x, y, t) & =e^{x-y}+\mathbb{N}^{-1}\left[\frac{u}{s} \mathbb{N}^{+}\left[v-w_{x} p_{y}-p_{x} w_{y}\right]\right] \\
w(x, y, t) & =e^{y-x}+\mathbb{N}^{-1}\left[\frac{u}{s} \mathbb{N}^{+}\left[w-p_{x} v_{y}-p_{y} v_{x}\right]\right] .
\end{aligned}
$$

In this stage, we assume an infinite series solutions of the unknown functions $p(x, y, t), v(x, y, t)$ and $w(x, y, t)$ of the form:

$$
\begin{aligned}
p(x, y, t) & =\sum_{n=0}^{\infty} p_{n}(x, y, t) \\
v(x, y, t) & =\sum_{n=0}^{\infty} v_{n}(x, y, t) \\
w(x, y, t) & =\sum_{n=0}^{\infty} w_{n}(x, y, t) .
\end{aligned}
$$

Then Eq. (5.23) can easily be re-written in the form:

$$
\begin{aligned}
& p(x, y, t)=e^{x+y}+\mathbb{N}^{-1}\left[\frac{u}{s} \mathbb{N}^{+}\left[\sum_{n=0}^{\infty} A_{n}(v, w)-\sum_{n=0}^{\infty} B_{n}(v, w)-\sum_{n=0}^{\infty} p_{n}(f)\right] 25\right) \\
& v(x, y, t)=e^{x-y}+\mathbb{N}^{-1}\left[\frac{u}{s} \mathbb{N}^{+}\left[\sum_{n=0}^{\infty} v_{n}-\sum_{n=0}^{\infty} C_{n}(w, p)-\sum_{n=0}^{\infty} D_{n}(w, p)\right]\right]
\end{aligned}
$$




$$
w(x, y, t)=e^{y-x}+\mathbb{N}^{-1}\left[\frac{u}{s} \mathbb{N}^{+}\left[\sum_{n=0}^{\infty} w_{n}-\sum_{n=0}^{\infty} E_{n}(p, v)-\sum_{n=0}^{\infty} F_{n}(p, v)\right]\right]
$$

Where $A_{n}, B_{n}, C_{n}, D_{n}, E_{n}$ and $F_{n}$ are Adomian polynomials which represent the nonlinear terms $v_{y} w_{x}, v_{x} w_{y}, w_{x} p_{y}, p_{x} w_{y}, p_{x} v_{y}$ and $p_{y} v_{x}$, respectively.

Therefore, from Eq. (5.25) we can easily generate the recursive relation as follows:

$$
\begin{aligned}
& p_{0}(x, y, t)=e^{x+y} \\
& p_{1}(x, y, t)=\mathbb{N}^{-1}\left[\frac{u}{s} \mathbb{N}^{+}\left[A_{0}(v, w)-B_{0}(v, w)-p_{0}\right]\right] \\
& p_{2}(x, y, t)=\mathbb{N}^{-1}\left[\frac{u}{s} \mathbb{N}^{+}\left[A_{1}(v, w)-B_{1}(v, w)-p_{1}\right]\right] .
\end{aligned}
$$

If we continue in this manner, we will eventually have:

$$
p_{n+1}(x, y, t)=\mathbb{N}^{-1}\left[\frac{u}{s} \mathbb{N}^{+}\left[A_{n}(v, w)-B_{n}(v, w)-p_{n}\right]\right] n \geq 0 .
$$

Similarly,

$$
\begin{aligned}
& v_{0}(x, y, t)=e^{x-y} \\
& v_{1}(x, y, t)=\mathbb{N}^{-1}\left[\frac{u}{s} \mathbb{N}^{+}\left[v_{0}-C_{0}(w, p)-D_{0}(w, p)\right]\right] \\
& v_{2}(x, y, t)=\mathbb{N}^{-1}\left[\frac{u}{s} \mathbb{N}^{+}\left[v_{1}-C_{1}(w, p)-D_{1}(w, p)\right]\right] .
\end{aligned}
$$

If we continue in the same manner, we will eventually have:

$$
v_{n+1}(x, y, t)=\mathbb{N}^{-1}\left[\frac{u}{s} \mathbb{N}^{+}\left[v_{n}-C_{n}(w, p)-D_{n}(w, p)\right]\right] n \geq 0 .
$$

Finally,

$$
\begin{aligned}
& w_{0}(x, y, t)=e^{-x+y} \\
& w_{1}(x, y, t)=\mathbb{N}^{-1}\left[\frac{u}{s} \mathbb{N}^{+}\left[w_{0}-E_{0}(p, v)-F_{0}(p, v)\right]\right] \\
& w_{2}(x, y, t)=\mathbb{N}^{-1}\left[\frac{u}{s} \mathbb{N}^{+}\left[w_{1}(p, v)-E_{1}(p, v)-F_{1}(p, v)\right]\right] .
\end{aligned}
$$

If we continue in the same manner, we will eventually have:

$$
w_{n+1}(x, y, t)=\mathbb{N}^{-1}\left[\frac{u}{s} \mathbb{N}^{+}\left[w_{n}-E_{n}(p, v)-F_{n}(p, v)\right]\right] n \geq 0
$$


Hence, from the recursive relation derived in Eq. (5.31), we can easily compute the remaining components of the unknown functions $p(x, y, t), v(x, y, t)$ and $w(x, y, t)$ as follows:

$$
\begin{aligned}
p_{1}(x, y, t) & =\mathbb{N}^{-1}\left[\frac{u}{s} \mathbb{N}^{+}\left[A_{0}(v, w)-B_{0}(v, w)-p_{0}\right]\right] \\
& =\mathbb{N}^{-1}\left[\frac{u}{s} \mathbb{N}^{+}\left[v_{0 y} w_{0 x}-v_{0 x} w_{0 y}-p_{0}\right]\right] \\
& =\mathbb{N}^{-1}\left[\frac{u}{s} \mathbb{N}^{+}\left[e^{x-y} e^{y-x}-e^{x-y} e^{y-x}-e^{x+y}\right]\right] \\
& =e^{x+y} \mathbb{N}^{-1}\left[\frac{u}{s} \mathbb{N}^{+}[1]\right] \\
& =-t e^{x+y} \\
v_{1}(x, y, t) & =\mathbb{N}^{-1}\left[\frac{u}{s} \mathbb{N}^{+}\left[v_{0}-C_{0}(w, p)-D_{0}(w, p)\right]\right] \\
& =\mathbb{N}^{-1}\left[\frac{u}{s} \mathbb{N}^{+}\left[v_{0}-w_{0 x} p_{0 y}-p_{0 x} w_{o y}\right]\right] \\
& =\mathbb{N}^{-1}\left[\frac{u}{s} \mathbb{N}^{+}\left[e^{x-y}-e^{y-x} e^{x+y}-e^{x+y} e^{y-x}\right]\right] \\
& =e^{x-y} \mathbb{N}^{-1}\left[\frac{u}{s} \mathbb{N}^{+}[1]\right] \\
& =t e^{x-y} \cdot \\
& =\mathbb{N}^{-1}\left[\frac{u}{s} \mathbb{N}^{+}\left[w_{0}-E_{0}(p, v)-F_{0}(p, v)\right]\right] \\
& =\mathbb{N}^{-1}\left[\frac{u}{s} \mathbb{N}^{+}\left[w_{0}-p_{0 x} v_{0 y}-p_{0 y} v_{o x}\right]\right] \\
& =\mathbb{N}^{-1}\left[\frac{u}{s} \mathbb{N}^{+}\left[e^{-x+y}-e^{x+y} e^{x-y}-e^{x+y} e^{x-y}\right]\right] \\
& =e^{x-y} \mathbb{N}^{-1}\left[\frac{u}{s} \mathbb{N}^{+}[1]\right] \\
& =t e^{-x+y}
\end{aligned}
$$

And

$$
\begin{aligned}
p_{2}(x, y, t)= & \mathbb{N}^{-1}\left[\frac{u}{s} \mathbb{N}^{+}\left[A_{1}(v, w)-B_{1}(v, w)-p_{1}\right]\right] \\
= & \mathbb{N}^{-1}\left[\frac{u}{s} \mathbb{N}^{+}\left[\left(v_{1 y} w_{0 x}+v_{0 y} w_{1 x}\right)-\left(v_{1 x} w_{0 x}+v_{0 x} w_{1 y}\right)-p_{1}\right]\right] \\
= & \mathbb{N}^{-1}\left[\frac { u } { s } \mathbb { N } ^ { + } \left[\left(t e^{x-y} e^{-x+y}+e^{x-y} t e^{-x+y}\right)\right.\right. \\
& \left.\left.--\left(t e^{x-y} e^{-x+y}+e^{x-y} t e^{-x+y}\right)+t e^{x+y}\right]\right]
\end{aligned}
$$




$$
\begin{aligned}
& =e^{x+y} \mathbb{N}^{-1}\left[\frac{u}{s} \mathbb{N}^{+}[t]\right] \\
& =e^{x+y} \mathbb{N}^{-1}\left[\frac{u^{2}}{s^{3}}\right] \\
& =\frac{t^{2} e^{x+y}}{2 !} \\
& v_{2}(x, y, t)=\mathbb{N}^{-1}\left[\frac{u}{s} \mathbb{N}^{+}\left[v_{1}-C_{1}(w, p)-D_{1}(w, p)\right]\right] \\
& =\mathbb{N}^{-1}\left[\frac{u}{s} \mathbb{N}^{+}\left[v_{1}-\left(w_{1 x} p_{0 y}+w_{0 x} p_{1 y}\right)-\left(p_{1 x} w_{0 y}+p_{0 x} w_{1 y}\right)\right]\right] \\
& =\mathbb{N}^{-1}\left[\frac { u } { s } \mathbb { N } ^ { + } \left[t e^{x-y}-\left(-t e^{-x+y} e^{x+y}+e^{-x+y} t e^{x+y}\right)\right.\right. \\
& \left.\left.-\left(-t e^{x+y} e^{-x+y}+e^{x+y} t e^{-x+y}\right)\right]\right] \\
& =e^{x-y} \mathbb{N}^{-1}\left[\frac{u}{s} \mathbb{N}^{+}[t]\right] \\
& =e^{x-y} \mathbb{N}^{-1}\left[\frac{u^{2}}{s^{3}}\right] \\
& =\frac{t^{2} e^{x-y}}{2 !} \text {. } \\
& w_{2}(x, y, t)=\mathbb{N}^{-1}\left[\frac{u}{s} \mathbb{N}^{+}\left[w_{1}-E_{1}(p, v)-F_{1}(p, v)\right]\right] \\
& =\mathbb{N}^{-1}\left[\frac{u}{s} \mathbb{N}^{+}\left[w_{1}-\left(p_{1 x} v_{0 y}+p_{0 x} v_{1 y}\right)-\left(p_{1 y} v_{0 x}+p_{0 y} v_{1 x}\right)\right]\right] \\
& =\mathbb{N}^{-1}\left[\frac { u } { s } \mathbb { N } ^ { + } \left[t e^{y-x}-\left(t e^{x+y} e^{x-y}-e^{x+y} t e^{x-y}\right)\right.\right. \\
& \left.\left.-\left(-t e^{x+y} e^{x-y}+e^{x+y} t e^{x-y}\right)\right]\right] \\
& =e^{-x+y} \mathbb{N}^{-1}\left[\frac{u}{s} \mathbb{N}^{+}[t]\right] \\
& =e^{y-x} \mathbb{N}^{-1}\left[\frac{u^{2}}{s^{3}}\right] \\
& =\frac{t^{2} e^{y-x}}{2 !} \text {. }
\end{aligned}
$$

Eventually, the approximate solution of the unknown functions $p(x, y, t), v(x, y, t)$ and $w(x, y, t)$ is given by:

$$
p(x, y, t)=\sum_{n=0}^{\infty} p_{n}(x, y, t)
$$




$$
\begin{aligned}
& =p_{0}(x, y, t)+p_{1}(x, y, t)+p_{2}(x, y, t)+\cdots \\
& =e^{x+y}-t e^{x+y}+\frac{t^{2} e^{x+y}}{2 !}+\cdots \\
& =e^{x+y}\left(1-t+\frac{t^{2}}{2 !}+\cdots\right) \\
& =e^{x+y-t}
\end{aligned}
$$

And

$$
\begin{aligned}
v(x, y, t) & =\sum_{n=0}^{\infty} v_{n}(x, y, t) \\
& =v_{0}(x, y, t)+v_{1}(x, y, t)+v_{2}(x, y, t)+\cdots \\
& =e^{x-y}+t e^{x-y}+\frac{t^{2} e^{x-y}}{2 !}+\cdots \\
& =e^{x-y}\left(1+t+\frac{t^{2}}{2 !}+\cdots\right) \\
& =e^{x-y+t}
\end{aligned}
$$

Lastly,

$$
\begin{aligned}
w(x, y, t) & =\sum_{n=0}^{\infty} w_{n}(x, y, t) \\
& =w_{0}(x, y, t)+w_{1}(x, y, t)+w_{2}(x, y, t)+\cdots \\
& =e^{y-x}+t e^{y-x}+\frac{t^{2} e^{y-x}}{2 !}+\cdots \\
& =e^{y-x}\left(1+t+\frac{t^{2}}{2 !}+\cdots\right) \\
& =e^{y-x+t}
\end{aligned}
$$

Hence, the exact solution of the unknown functions in the given system of nonlinear coupled partial differential equation is given by:

$$
\begin{aligned}
p(x, y, t) & =e^{x-y+t} \\
v(x, y, t) & =e^{x-y+t} \\
w(x, y, t) & =e^{y-x+t} .
\end{aligned}
$$

This is in agreement with the results obtained by ADM [18] and LDM [25]. 


\section{Conclusion}

In this article, the Natural Decomposition Method (NDM) was proposed for solving system of nonlinear coupled partial differential equation with initial conditions. We successfully found an exact solutions in all the two examples and compare the result with ADM [1] and [18], LDM [25], HAM [26] and VIM [27]. This clearly shows that the Natural Decomposition Method NDM introduces a significant improvement in the fields over existing techniques. Our goal in the future is to apply the NDM to other nonlinear PDEs which arise in other areas of applied science.

\section{Acknowledgments}

The authors would like to thank the Editor and the anonymous referees' for their comments and suggestions on this paper.

\section{References}

[1] G. Adomian, Solving frontier problems of physics: the decomposition method, Kluwer Acad. Publ (1994).

[2] G. Adomian, A new approach to nonlinear partial differential equations, J. Math. Anal. Appl., 102, (1984), 420-434.

[3] Tarig M. Elzaki, The New Integral Transform "Elzaki" Transform, Global Journal of Pure and Applied Mathematics, ISSN 0973-1768, 1, (2011), 5764.

[4] Tarig M. Elzaki, Salih M. Elzaki, Application of New Transform "Elzaki Transform" to Partial Differential Equations, Global Journal of Pure and Applied Mathematics, ISSN 0973-1768, 1, (2011), 65-70.

[5] Tarig M. Elzaki, Salih M. Elzaki, On the Connections Between Laplace and Elzaki transforms, Advances in Theoretical and Applied Mathematics, 0973-4554, 6, Number 1, (2011), 1-11.

[6] Tarig M. Elzaki, Salih M. Elzaki, On the Elzaki Transform and Ordinary Differential Equation with Variable Coefficients, Advances in Theoretical and Applied Mathematics, ISSN 0973-4554, 6, Number 1, (2011), 13-18. 
[7] Tarig M. Elzaki, Adem Kilicman and Hassan Eltayeb, On Existence and Uniqueness of Generalized Solutions for a Mixed-Type Differential Equation, Journal of Mathematics Research, 2, No. 4, (2010), 88-92.

[8] Tarig M. Elzaki, Existence and Uniqueness of Solutions for Composite Type Equation, Journal of Science and Technology, (2009), 214-219.

[9] F.B.M. Belgacem, A.A. Karaballi and S.L. Kalla, Analytical investigations of the Sumudu transform and applications to integral production equations, Mathematical Problems in Engineering, 3, (2003), 103-118.

[10] F.B.M. Belgacem, A.A. Karaballi, Sumudu transform fundamental properties, investigations and applications, Journal of Applied Mathematics and Stochastic Analysis, 40, (2006), 1-23.

[11] F.B.M. Belgacem, Introducing and analyzing deeper Sumudu properties, Nonlinear Studies Journal, 13, No. 1, (2006), 23-41.

[12] F.B.M. Belgacem, R. Silambarasan, Maxwell's equations by means of the natural transform, Mathematics in Engineering, Science and aerospace, 3, No. 3, (2012), 313-323.

[13] F.B.M. Belgacem, Sumudu transform applications to Bessel's functions and equations, Applied Mathematical Sciences, 4, No. 74, (2010), 36653686.

[14] F.B.M. Belgacem, Sumudu applications to Maxwell's equations, PIERS Online, 5, No. 4, (2009), 355-360.

[15] F.B.M. Belgacem, Applications of Sumudu transform to indefinite periodic parabolic equations, Proceedings of the 6th International Conference on Mathematical Problems and Aerospace Sciences, (ICNPAA 06), Chap. 6, 5160, Cambridge Scientific Publishers, Cambridge, UK (2007).

[16] F.B.M. Belgacem, R. Silambarasan, Theoretical investigations of the natural transform, Progress In Electromagnetics Research Symposium Proceedings, Suzhou, China, Sept. 12 16, (2011).

[17] F.B.M. Belgacem, R. Silambarasan, Maxwell's equations solutions through the natural transform, Mathematics in Engineering, Science and Aerospace 3, No. 3, (2012), 313-323. 
[18] M.G.M. Hussain, F.B.M. Belgacem, Transient solutions of Maxwell's equations based on Sumudu transform, Progress in Electromagnetics Research, 74, (2007), 273-289.

[19] Z.H. Khan, W.A. Khan, N-transform properties and applications, NUST Jour. of Engg. Sciences, 1, No. 1, (2008), 127-133.

[20] M. Rawashdeh, Improved Approximate Solutions for Nonlinear Evolutions Equations in Mathematical Physics Using the RDTM, Journal of Applied Mathematics and Bioinformatics, 3, No. 2, (2013), 1-14.

[21] M. Rawashdeh, Using the Reduced Differential Transform Method to Solve Nonlinear PDEs Arises in Biology and Physics, World Applied Sciences Journal, 23, No. 8, (2013), 1037-1043.

[22] M. Rawashdeh, N. Obeidat, On Finding Exact and Approximate Solutions to Some PDEs Using the Reduced Differential Transform Method, Applied Mathematics and Information Sciences, 8, No. 5, (2014), 1-6.

[23] M.R. Spiegel, Theory and Problems of Laplace Transforms, Schaums Outline Series, McGraw-Hill, New York (1965).

[24] A.M. Wazwaz, Partial Differential Equations and Solitary Waves Theory, Springer-Verlag, Heidelberg (2009).

[25] S.T. Khuri, A Laplace decomposition algorithm applied to a class of nonlinear partial differential equations, Journal of Applied Mathematics, 1, (2001), 141-155.

[26] A.K. Alomari, M.S.M. Noorani and R. Nazar, The homotopy analysis method for the exact solution of the $K(2,2)$ and Coupled Burgers equations, Applied Mathematical Sciences, 2, (2008), 1963-1977.

[27] M.A. Abdou, A.A. Soliman, Variational iteration method for solving Burger's and Coupled Burger's equations, Journal of Computational and Applied Mathematics, 181, (2005), 245-251. 